\title{
Interplay between coherent and dissipative dynamics of bosonic doublons in an optical lattice
}

\author{
M. J. Mark $\odot,{ }^{1,2}$ S. Flannigan $\odot,{ }^{3}$ F. Meinert $\odot,{ }^{4}$ J. P. D’Incao $\odot,{ }^{5}$ A. J. Daley $\odot,{ }^{3}$ and H.-C. Nägerl $\odot^{1}$ \\ ${ }^{1}$ Institut für Experimentalphysik und Zentrum für Quantenphysik, Universität Innsbruck, 6020 Innsbruck, Austria \\ ${ }^{2}$ Institut für Quantenoptik und Quanteninformation, Österreichische Akademie der Wissenschaften, 6020 Innsbruck, Austria \\ ${ }^{3}$ Department of Physics \& SUPA, University of Strathclyde, Glasgow, G4 ONG, Scotland, United Kingdom \\ ${ }^{4}$ 5. Physikalisches Institut and Center for Integrated Quantum Science and Technology, Universität Stuttgart, Pfaffenwaldring 57, \\ 70569 Stuttgart, Germany \\ ${ }^{5}$ JILA, National Institute of Standards and Technology, and the University of Colorado, Department of Physics, Boulder, Colorado 80309, USA
}

(Received 19 May 2020; accepted 8 September 2020; published 8 October 2020)

\begin{abstract}
We observe the dissipative dynamics of a dense, strongly interacting gas of bosonic atom pairs in an optical lattice, controlling the strength of the two-body interactions over a wide parameter regime. We study how threebody losses contribute to the lattice dynamics, addressing a number of open questions related to the effects of strong dissipation in a many-body system, including the relationship to the continuous quantum Zeno effect. We observe rapid break-up of bound pairs for weak interactions, and for stronger interactions we see doublon decay rates that are asymmetric when changing from attractive and repulsive interactions and which strongly depend on the interactions and on-site loss rates. By comparing our experimental data with a theoretical analysis of few-body dynamics, we show that these features originate from a nontrivial combination of dissipative dynamics described by a lattice model beyond a standard Bose-Hubbard Hamiltonian, and the modification of three-atom dynamics on a single site, which is generated alongside strong three-body loss. Our results open new possibilities for investigating bosonic atoms with strong three-body loss features and allow for the better understanding of the parameter regimes that are required to realize strong effective three-body interactions.
\end{abstract}

DOI: 10.1103/PhysRevResearch.2.043050

\section{INTRODUCTION}

Ultracold atomic gases in optical lattices provide a platform for investigating novel many-body coherent and dissipative dynamics [1-5]. In particular, processes such as collisional loss of atoms, which we often seek to avoid, can exhibit features such as the continuous quantum Zeno effect [6-9], where a strong dissipative process can prevent coherent dynamics from taking place $[10,11]$. This has resulted in proposals to realize effective three-body interactions through three-body loss [12-15]. However, there are important open questions related to how these dynamics work when the loss rates become comparable to or larger than the energy band gap, rendering a standard Bose-Hubbard (BH) description insufficient. In this work, we explore the interplay between coherent and dissipative dynamics for a gas of bosonic atom pairs, comparing the dependence of the three-body loss on the interaction strength to theoretical models beyond the standard BH Hamiltonian that combine lattice dynamics with a careful treatment of on-site three-body dynamics that renormalizes the coefficients of coherent interactions and loss. Understanding these effects across different regimes provides a path for

Published by the American Physical Society under the terms of the Creative Commons Attribution 4.0 International license. Further distribution of this work must maintain attribution to the author(s) and the published article's title, journal citation, and DOI. future studies of exotic quantum phases induced by strong local three-body loss [16-23].

We make use of the control available in optical lattice systems and study interacting cesium atoms in the vicinity of a broad Feshbach resonance [24], which allows us to tune the $s$-wave scattering length $a_{S}$ and therefore the interactions from weak to strong and from repulsive $\left(a_{S}>0\right)$ to attractive $\left(a_{S}<0\right)$. The two-body properties of our system are well understood [25-27] and it is straightforward to prepare an initial state with on-site pairs (often referred to as doublons) $[28,29]$. Doublons are in general stable in optical lattice systems because of the lack of dissipative phonon modes that can remove energy on short timescales [28,30]. However, by increasing the interaction strength one enters a regime where the energy associated with strong three-body dissipative processes $[31,32]$ can easily exceed the band gap in the lattice. This raises questions on how to treat this system with an effective BH model [25,26,33-35], including the question whether off-site loss mechanisms become important in understanding the resulting dynamics and how effective model parameters would need to be rescaled in that case.

Below, we investigate the effects of strong three-body losses starting with doubly occupied sites and find unexpected phenomena arising from an off-resonant loss process where a single particle tunnels into a virtual triply occupied site. For strong attractive interactions, we observe an interesting nonlinear dependence of the doublon decay rates on $a_{S}$ and attempt to model this with a BH-type model resulting in values for decay rates that are too large if nearest-neighbor losses are 

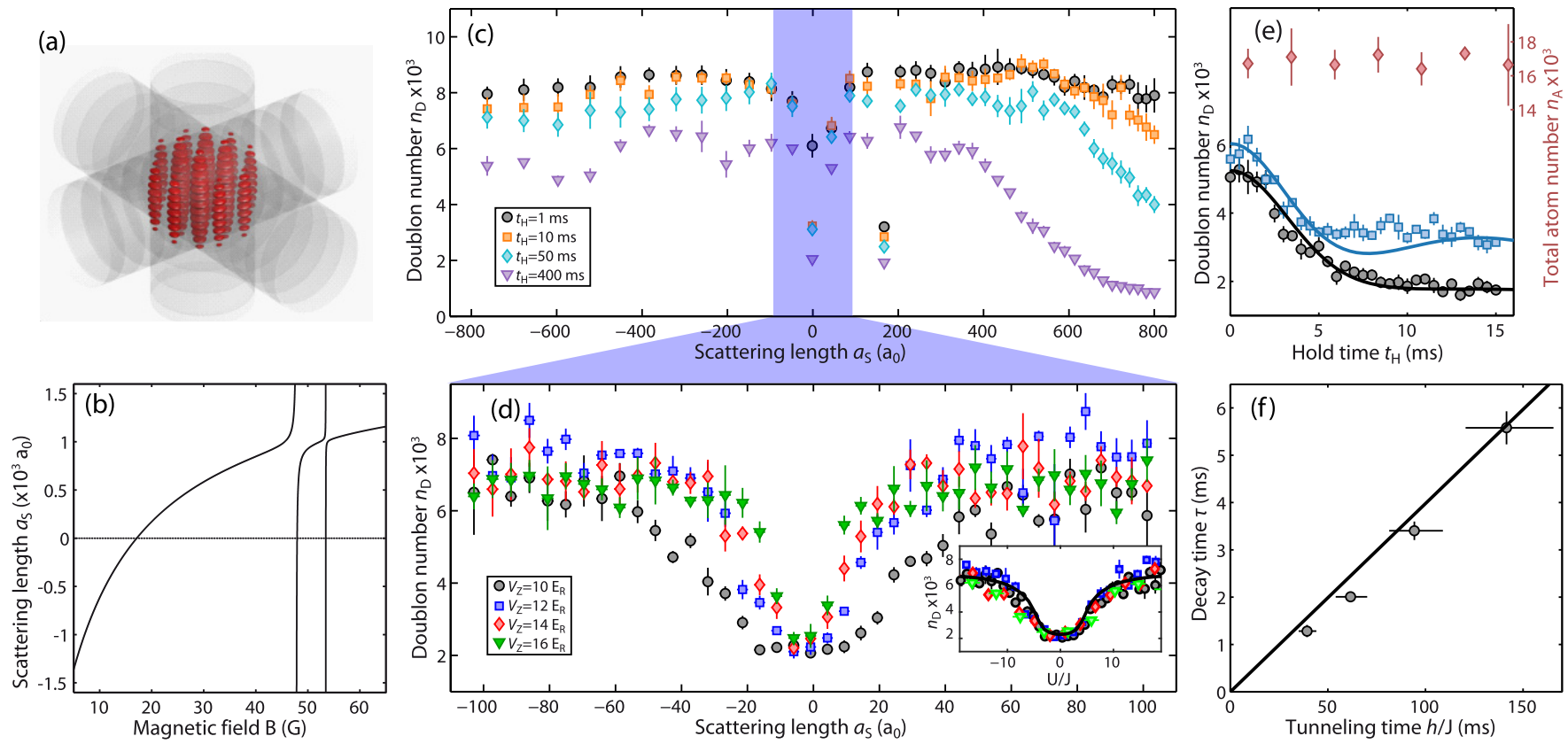

FIG. 1. (a) Illustration of the lattice system. (b) Dependence of $a_{S}$ on the magnetic offset field $B$. (c) Number of doublons $n_{D}$ as a function of $a_{S}$ for $t_{H}$ as indicated for $V_{x, y, z}=(30,30,10) E_{R}$. The loss feature near $a_{S}=180 \mathrm{a}_{0}$ results from a narrow Feshbach resonance. (d) Number of doublons $n_{D}$ as a function of $a_{S}$ for $t_{H}=100 \mathrm{~ms}$ with $V_{z}$ as indicated. The inset plots the same data as a function of $U / J$, with the solid line showing the numerical result (see text). (e) Number of doublons $n_{D}$ as a function of $t_{H}$ for $U / J \approx 0$ (circles) and $U / J=3.5$ (squares) at $V_{z}=14 E_{R}$ and $V_{x, y}=30 E_{R}$. The total atom number $n_{A}$ (diamonds) for $U / J \approx 0$ is also shown as a reference. The solid lines are the corresponding numerical results. (f) Decay time $\tau$, obtained from exponential fits to the decay curves for $U / J \approx 0$ for different $V_{z}$, as a function of the calculated tunneling time $h / J$. The solid line is the estimated decay time $\tau=\hbar /(4 J)$. The vertical and horizontal error bars reflect the one-sigma statistical error and a $5 \%$ uncertainty in $V_{z}$, respectively.

included but too low if they are simply ignored. This indicates that the mixing with higher-energy bands is causing a renormalization of both the off-site and the on-site coefficients. When further increasing the attractive interaction strength, we observe a decrease on the decay rate similar to a quantum Zeno type suppression [11]. However, through first-principle calculations of the on-site losses induced through mixing with three-body Efimov states [31,32,36-38], we find that on-site loss rates are too low to induce this type of suppression which instead suggests that the decrease of the decay rates is due to an additional renormalization of the terms responsible for the coherent dynamics.

\section{EXPERIMENTAL SEQUENCE}

Our experiments start with the production of an essentially pure Bose-Einstein condensate (BEC) of $1.0 \times 10^{5} \mathrm{Cs}$ atoms in the lowest hyperfine state in a crossed optical trap [39]. To prepare a comparatively dilute system of doublons, we nonadiabatically load the BEC within 2 ms into a cubic optical lattice formed by three retroreflected laser beams with a wavelength of $\lambda=1064.5 \mathrm{~nm}$, see Fig. 1(a). Initially, the lattice depths along all three directions are set to $V_{x, y, z}^{0}=30 E_{R}$, where $E_{R}=h^{2} /\left(2 m \lambda^{2}\right)$ is the recoil energy with the mass $m$ of the Cs atom. During lattice loading $a_{S}$ is set to $220 \mathrm{a}_{0}$, where $\mathrm{a}_{0}$ is Bohr's radius. The doublon sample is then purified by Feshbach molecule formation, cleaning, and dissociation [40], see also Appendix A.
Next, we tune $a_{S}$ to a value between $-800 \mathrm{a}_{0}$ and $+800 \mathrm{a}_{0}$ by ramping the offset magnetic field $B$ with a rate of $\sim 2.5 \mathrm{G} / \mathrm{ms}$ to a value between about $8 \mathrm{G}$ and $37 \mathrm{G}$, exploiting a broad Feshbach resonance, see Fig. 1(b) [41]. Note that the tuning range includes a zero crossing for $a_{S}$ located at 17.119 G. To induce dynamics of the doublons, we quench the lattice depth along the $z$ direction within $1 \mathrm{~ms}$ to a new value $V_{z}$, realizing nearly isolated one-dimensional (1D) $\mathrm{BH}$ systems. After a variable hold time $t_{H}$ we interrupt the dynamics by raising $V_{z}$ in $1 \mathrm{~ms}$ back to its original value. Finally, we determine the remaining number of doublons by employing the association-cleaning-dissociation procedure a second time before detecting the atom number after a short time-of-flight.

\section{WEAK INTERACTION REGIME}

The doublon number $n_{D}$ shows a strong dependence on $a_{S}$ as can be seen in Fig. 1(c) for four different hold times $t_{H}$. Clearly, our data exhibits a pronounced asymmetry between strongly attractive and strongly repulsive interactions. We first focus on the region near the zero crossing. A close-up of this region is presented in Fig. 1(d) for four different values of $V_{z}$ and a hold time $t_{H}=100 \mathrm{~ms}$ for which the dynamics has reached a steady state. A pronounced dip for $n_{D}$ centered at $a_{S}=0$ is evident. In this regime of comparatively weak interactions a standard lowest-band BH description [42] should be 
valid. Explicitly the standard Bose-Hubbard model is $(\hbar=1)$

$$
H_{\mathrm{BH}}=-J \sum_{\langle i, j\rangle} b_{i}^{\dagger} b_{j}+\frac{U}{2} \sum_{i} b_{i}^{\dagger 2} b_{i}^{2},
$$

where $J$ is the nearest-neighbor tunneling and $U$ is the onsite two-body interaction. The dip, which is essentially symmetric as a function of $a_{S}=0$, results from rapid dissociation of the doublons when the $\mathrm{BH}$ interaction energy $|U|$ and the tunneling energy $J$ become comparable, irrespective of the sign of $U$, while for the regime $|U| \gg J$ doublons are bound together and their number stays constant. Indeed, when plotting the data as a function of $U / J$ we observe a collapse onto a single curve, see inset to Fig. 1(d). Note that $n_{D}$ remains nonzero at $U / J=0$, which is a consequence of the finite size of the 1D systems, resulting in a nonzero probability to find two atoms on the same lattice site.

We stress that in this regime of comparatively weak interactions, the doublons dissociate but atoms are not lost from the sample. This is evident from Fig. 1(e), which depicts the dissociation dynamics for two values of $U / J$. While the total atom number stays constant, we observe a rapid decay for $n_{D}$ with a rate independent of $U / J$ toward the steady-state doublon number shown in Fig. 1(d). Moreover, the initial decay time $\tau$, obtained from an exponential fit to the data, provides a direct measure of $J$ and obeys the linear relation $\tau=\frac{\hbar}{4 J}$, see Fig. 1(f).

So far, our observations near $a_{S}=0$ are all captured by the standard lowest-band BH model. To demonstrate this, we numerically calculate the dynamics by exact diagonalization, see Appendix B for details. Comparison with the experimental data as shown in Fig. 1(e) gives good agreement. The small deviations probably arise from finite-size effects. Also, the steady-state doublon number is well captured, see the inset to Fig. 1(d).

\section{STRONG INTERACTION REGIME}

Let us now turn to the unexplored regime of strong interactions, where the standard BH description no longer applies. As evident from Fig. 1(c), we observe increased loss of doublons with increasing positive $a_{S}$ but not so for negative $a_{S}$. Figure 2(a) shows the typical time evolution of $n_{D}$ for strong repulsive interactions, along with the number of singly occupied sites $n_{S}$ and the total atom number $n_{A}$. In contrast to the case of weak interactions, the loss of double occupancy is caused by a true loss of atoms from the system. We observe that the number of singly occupied sites increases until all doublons are lost. The ratio of lost doublons to lost atoms is 2.1(2):3, the ratio of lost doublons to reappearing atoms at singly occupied sites is $2.1(3): 1$. This represents strong evidence that the dominant loss process is three-body recombination, where two neighboring doublons exhibit a small probability to decay via a triply occupied site, leaving one singly occupied site behind. Note that this mechanism differs from the elastic decay observed for doublons composed of fermions [29].

For a quantitative comparison with a theoretical model, we perform the same experiment in a dense system, i.e., starting from a Mott shell with predominant double occupancy,
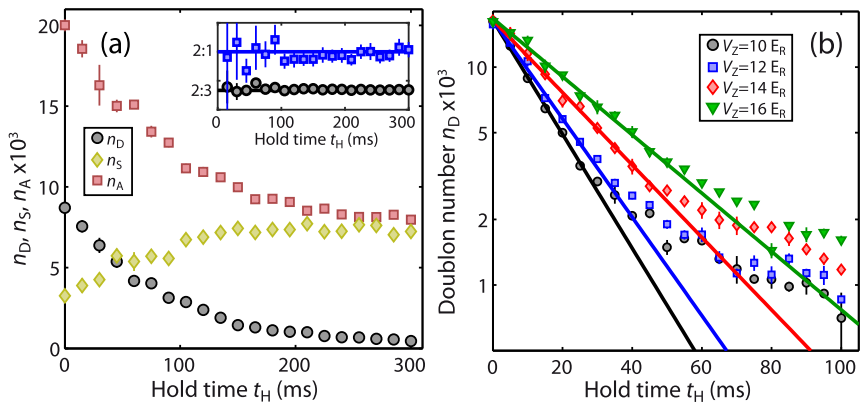

FIG. 2. (a) Doublon decay for strong interactions: Number of doublons $n_{D}$ (circles), singly occupied sites $n_{S}$ (diamonds) and total atom number $n_{A}$ (squares) as a function of hold time $t_{H}$ for a dilute sample for $a_{S}=800 \mathrm{a}_{0}$ and $V_{z}=14 E_{R}$. The inset shows the ratio of lost doublons to lost atoms (circles) and reappearing singly occupied sites (squares). (b) Doublon decay for strong interactions and high density: Number of doublons $n_{D}$ as a function of $t_{H}$ for $a_{S}=800 \mathrm{a}_{0}$ for $V_{z}$ as indicated. The solid lines are fits by an exponential decay to the datapoints with more than $50 \%$ of the initial value, giving decay times $\tau=16.6(0.7), 19.2(1.1), 26.1(0.8)$, and 32.5(1.3) ms.

cleaned from singly occupied sites in the same way as before. This ensures that tunneling dynamics of the doublons themselves do not play any role in the initial dynamics. We estimate the filling fraction to be close to unity for an initial length of about 20 sites for the 1D systems. Figure 2(b) depicts typical decay curves for $n_{D}$ from which we deduce initial doublon decay rates $\gamma_{D}$ from exponential fits to the data. The decay rates slow down with increased loss as the system gets more dilute. We hence use only the initial parts of the loss curves for the fit.

In this way, we measure $\gamma_{D}$ for different values of $a_{S}$ and $V_{z}$. Figure 3(a) shows $\gamma_{D}$ for a very broad range of interaction strength, from $-2000 \mathrm{a}_{0}$ to $+800 \mathrm{a}_{0}$, revealing the drastic asymmetry between repulsive and attractive interactions that could already be seen in Fig. 1(c). Note that we limit the hold time to $400 \mathrm{~ms}$ to avoid tunneling dynamics between neighboring 1D systems. This, however, leads to comparatively large uncertainties for small decay rates.

\section{THEORETICAL DESCRIPTION}

We model this behavior by an extended BH model that accounts for on-site, $U$, and nearest-neighbor, $\tilde{U}$, two-body interactions as well as dissipative on-site three-body loss, $\gamma_{3}$,

$$
\begin{aligned}
H= & H_{\mathrm{BH}}+\tilde{U} \sum_{i}\left[b_{i}^{\dagger} b_{i}^{\dagger} b_{i} b_{i+1}+b_{i}^{\dagger} b_{i+1}^{\dagger} b_{i+1} b_{i+1}+\text { H.c. }\right] \\
& -i \frac{\gamma_{3}}{12} \sum_{i} b_{i}^{\dagger 3} b_{i}^{3}
\end{aligned}
$$

The coefficients of this model are functions of the scattering length, $a_{s}$, but are also given in terms of the Wannier functions which we present explicitly in Appendix C. Note that we neglect terms corresponding to site-to-site three-body loss. For large values of $\left|a_{S}\right|$, one might expect to obtain large contributions from off-site loss processes. However, this picture only holds for sufficiently small loss rates compared to the band separation energy when the lowest-band Wannier 

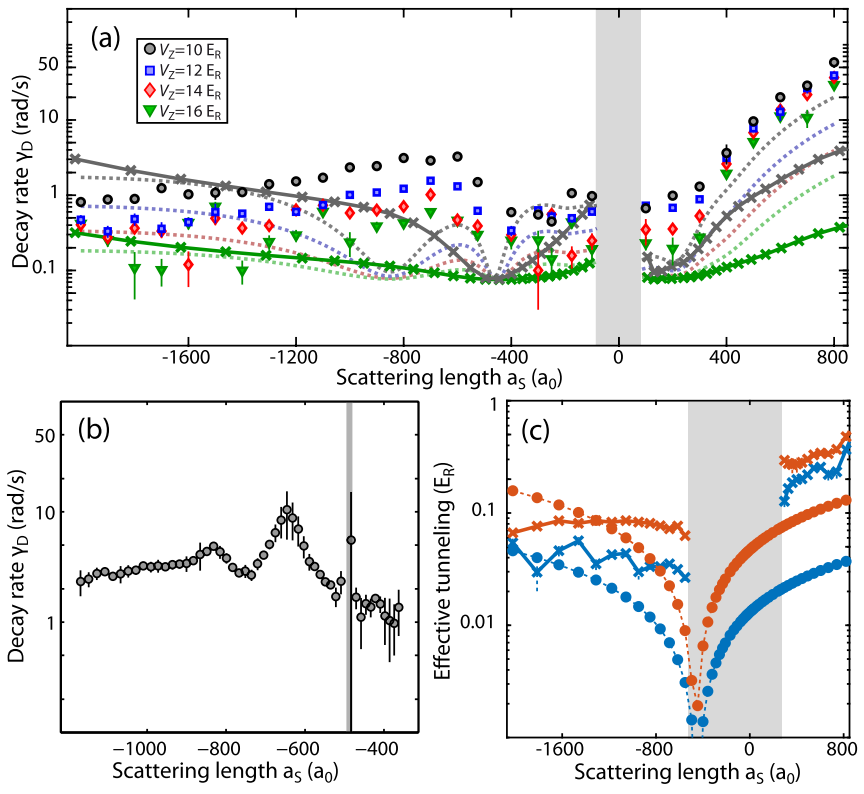

FIG. 3. Decay rates for strong interactions and high density. (a) Doublon decay rate $\gamma_{D}$ starting from the two-atom Mott shell for the full interaction range with $V_{z}$ as indicated plotted against $\mathrm{a}_{S}$. Dotted lines (solid lines with crosses) are predictions without (with) modified on-site parameters (see text). Line colors are chosen to match the corresponding experimental data. The shaded area indicates the region of weak interactions where $U \gg J$ is not fulfilled. (b) Fine scan of $\gamma_{D}$ for attractive interactions in the range from $-1100 \mathrm{a}_{0}$ to $-400 \mathrm{a}_{0}$ with $V_{z}=10 E_{R}$. The shaded area indicates the presence of a narrow Feshbach resonance. (c) Renormalized tunneling coefficient within the extended BH model (dotted line with filled circles) and determined from the experimental data (solid line with crosses) for $V_{z}=10$ (red) and $V_{z}=16$ (blue). The shaded area denotes the region where the perturbative treatment for the latter is not valid.

functions provide a good local basis. In the presence of large loss rates, as is the case for large $\left|a_{S}\right|$, the Wannier states mix with states from higher bands and we find that the initial overlaps of nearest-neighboring wave functions lead to a very rapid initial decay, although only resulting in a very small loss of atoms, see Appendix E. This happens on timescales much faster than the experimental measurements, resulting in renormalized states that are more localized on each lattice site. Atom loss is then driven by on-site decay only, combined with nonresonant tunneling processes, including density-assisted tunneling from nearest-neighbor interactions.

To understand the importance of competing on-site and tunneling processes in the dynamics of particle loss, we perform calculations in which we evolve the initial doubly occupied Mott state using a Lindblad master equation. We incorporate three-body loss and also single-particle loss terms that account for residual heating induced by the lattice light and/or background gas collisions [26]. We extract the decay rate $\gamma_{D}$ from an exponential fit to the computed doublon density. In order to identify the roles of different processes, we use two complementary methods to estimate coefficients for our model.
In the first approach, we calculate lattice coefficients using single-particle Wannier functions associated with the lowest Bloch band. On-site loss is modeled as a zero-range three-body recombination with a rate coefficient $L_{3}$, with parameters for Cs fitted to the experimental data from Ref. [24]. As we will see below, the resulting model predicts decay rates at the same order of magnitude as the experimental measurements, capturing qualitative aspects well for positive scattering lengths. However, it fails to predict the finer details for negative scattering lengths due to the presence of a large three-body resonance, for which we clearly must go beyond a standard $\mathrm{BH}$ model description.

In our second approach, we determine more accurate onsite parameters by taking the lowest on-site three-body energy level obtained by using the hyperspherical adiabatic representation $[31,37]$. This allows us to calculate, from first principles, the on-site three-body losses from and energy shifts to the lowest band due to three-body interactions mixing with higher bands [36]. For on-site two-body interactions we also take into account mixing with higher bands using the result presented in Ref. [43] for a single harmonic trap. We find that by incorporating these corrections from strong interactions the main features near the resonance are better approximated. However, we do not modify the nearest-neighbor tunneling and interaction terms and find that these must also play an important role away from the resonance.

\section{RESULTS AND COMPARISON}

In Fig. 3(a) we compare $\gamma_{D}$ obtained from the two approaches with the experimental data. We find that for repulsive interactions the decay rates increase with increasing $a_{S}$, in accordance with the predictions. We also find that the first approach (dotted lines) predicts a minimum in the decay rate around $a_{\mathrm{S}}=-800 a_{0}$, which coincides with the strong Efimov resonance reported in $L_{3}$ [24] and which is not observed in our lattice experiment. Our second theoretical approach (solid lines with symbols), incorporating the effects of the lattice potential on the loss rates, predicts a suppression of the $L_{3}$ resonance for negative scattering lengths and we obtain values for $\gamma_{D}$ that qualitatively match the experiment. This peak suppression is due to the interplay between the Efimov resonance, the mixing with higher bands and the lattice potential, which creates a spread of the particles in momentum space. Indeed, this spread is of the same magnitude as the temperature-induced energy spread that was found to suppress the Efimov resonance in previous measurements [24,44]. In addition, effects of mixing with higher excited states introduce further modifications to the on-site, but also the off-site, loss coefficients. The ratio of the corrections of the onsite terms to the nearest-neighbor terms will have a complex dependence on the scattering length leading to surprising features in the decay rate dependence in Fig. 3(b), such as the double peak feature. However, a detailed understanding of this will require future analysis on the renormalization of the nearest-neighbor terms.

For positive scattering lengths, the calculation with the renormalized onsite coefficients (solid lines with symbols) leads to an even larger discrepancy compared to the naive calculation with the bare coefficients in the lowest band (dotted 
lines). This is a strong indication that in this regime there are also important corrections to the nearest-neighbor tunneling processes arising from the mixing with states in higher-energy bands. Additionally, both approaches predict a much larger dependence on the trapping potential than is experimentally observed, indicating that the renormalization effects may be stronger for tighter trapping potentials.

Furthermore, including corrections from strong interactions [solid lines with symbols in Fig. 3(a)] predicts that, for increasingly large attractive interactions, the decay rates also increase, in sharp contrast to the experimental data. We initially interpreted the trend in the measurements to be due to a quantum-Zeno-type suppression of the losses [11], but the values of the renormalized coefficients in our calculation predict on-site loss rate values that are too low for this to occur (see Appendix D).

Instead, we believe that this trend can be accounted for through the incorporation of two effects not currently taken into account in our model: mixing between ground and excited on-site three-body states (see Appendix D), and a further renormalization of the effective tunneling elements [27]. Our experiment enables us to estimate what the values of the rescaled tunneling coefficients, $\tilde{J}=|3 \sqrt{6} \tilde{U}-\sqrt{6} J|$ (see Appendix $C$ ), would need to be to produce the measured decay rates, accounting for interaction-modified tunneling coefficients. These are shown in Fig. 3(c) and predict a strong enhancement of the tunnelings rates for positive scattering lengths. For negative $a_{S}$ there is a saturation of the magnitudes of the rescaled tunneling rate, an effect that is more pronounced for shallow lattices. This analysis shows that, we can drastically improve the quantitative agreement between the calculations and measurements with only a relatively small variation of the tunneling terms. This simple estimate and the calculation of the renormalized onsite loss coefficients, indicate that the reduction in the overall decay rate is not due to a continuous quantum Zeno type suppression but instead arises from slower coherent dynamics induced by the strong onsite losses mixing with higher-energy states. This then has implications for future experiments attempting to realize the novel many-body phases induced by strong three-body losses [12-15].

\section{CONCLUSION AND OUTLOOK}

In summary, we have investigated the dynamics of bosonic doublons for a broad range of attractive and repulsive interactions. We have found that for strong two-body interactions (attractive and repulsive) the dynamics are dominated by offresonant decay processes induced by on-site three-body loss, with a large renormalization of the coefficients in both the dissipative and coherent processes, which can be understood by solving the three-body problem on-site. For particularly strong interactions, we enter a regime, where strong interactions lead to further corrections, which can be attributed to a combination of modified effective tunneling rates, and additional mixing of on-site three-body eigenstates.

In this way, our experiments and theoretical analysis both answer existing and (for stronger interactions) open new questions on the most accurate means to model dissipation for atoms in optical lattices. This is important for high-efficiency molecular formation [45] but also opens routes to further explore physics beyond the Bose-Hubbard model, and as well as to identify regimes of true quantum Zeno effect suppression of three-body occupation. This opens routes toward a range of exotic many-body states driven by three-body projections.

\section{ACKNOWLEDGMENTS}

We thank Suzanne McEndoo for important discussions during the early stages of this work. The Innsbruck team gratefully acknowledges funding by the European Research Council (ERC) under Project No. 278417 and under Project No. 789017 and by the Austrian Science fund (FWF) via the FWF-ANR joint project with Project No. I2922N36. This work was supported in part by the European Union's Horizon 2020 research and innovation program under Grant No. 817482 PASQuanS. S.F. acknowledges the financial support of the Carnegie trust. J.P.D. acknowledges support from National Aeronautics and Space Administration (NASA/JPL 1502690). F.M. acknowledges support by the Carl Zeiss Foundation via IQST and is indebted to the Baden-Württemberg-Stiftung for the financial support by the Eliteprogramm for Postdocs.

\section{APPENDIX A: EXPERIMENTAL PREPARATION AND DETECTION OF DOUBLONS}

A pure sample of doubly occupied sites as well as the detection of only double occupancy is accomplished via a sequence of molecule association, cleaning, and dissociation. First, atom pairs on a lattice site are associated to weakly bound molecules by crossing a narrow $g$-wave Feshbach resonance with a pole at $19.8 \mathrm{G}$. The system is afterward cleaned from remaining singly occupied sites by combining a rapid adiabatic passage transferring atoms from $\left|F=3, m_{F}=3\right\rangle$ to $\left|F=4, m_{F}=4\right\rangle$ using a microwave field with a resonant lightpulse. The Feshbach molecules are left untouched during this process. After dissociating the molecules again we detect the number of atoms with standard absorption imaging. The overall efficiency of doublon detection has been calibrated to be $80(3) \%$. Single occupancy is measured by associating doubly occupied sites into Feshbach molecules and increasing their binding energy via magnetic field ramping. This procedure efficiently hides them during the imaging process, enabling the detection of only the remaining singly occupied sites. The overall efficiency of singly occupation detection is $92(2) \%$, limited by residual losses of singly occupied sites caused by the magnetic field ramp across the Feshbach resonance for molecule association.

\section{APPENDIX B: EXACT DIAGONALIZATION STANDARD BH MODEL}

We perform exact diagonalization of small 1D systems of typically 6 atoms on 10-11 lattice sites with hard wall boundary conditions. To mimic the dilute sample of randomly distributed doublons, we compute the time evolution of each possible initial doublon state and average the time evolution of the doublon probability. To extract the equilibrium value 
after the initial dynamics, we compute the median value in a time frame between 50 and $100 \mathrm{~J}$.

\section{APPENDIX C: EXTENDED BH MODEL}

The $1 \mathrm{D}$ extended $\mathrm{BH}$ model that we use consists of onsite and nearest-neighbor two-body interactions and on-site three-body loss. In principle there can also be three-body recombination effects between atoms in nearest-neighboring Wannier states. However, we obtain much better agreement with the experiment for negative values for $a_{S}$ if we neglect these contributions. This makes physical sense in the limit where the three-body loss rate dominates the dynamics, as any small initial overlap between neighboring states would be very rapidly lost, on timescales much shorter than the experimentally measured dynamics, resulting in more localized on-site wave functions. Explicitly, the Hamiltonian is given by $(\hbar=1)$,

$$
\begin{aligned}
H= & -J \sum_{\langle i, j\rangle} b_{i}^{\dagger} b_{j}+\frac{U}{2} \sum_{i} b_{i}^{\dagger 2} b_{i}^{2} \\
& +\tilde{U} \sum_{i}\left[b_{i}^{\dagger} b_{i}^{\dagger} b_{i} b_{i+1}+b_{i}^{\dagger} b_{i+1}^{\dagger} b_{i+1} b_{i+1}+\text { H.c. }\right] \\
& -i \frac{\gamma_{3}}{12} \sum_{i} b_{i}^{\dagger 3} b_{i}^{3} .
\end{aligned}
$$

The coefficients, calculated through the Wannier functions associated to the lowest Bloch band centered at position $z_{i}$ in the longitudinal direction, $w\left(z-z_{i}\right)$, and $\vec{r}_{i}$ in the radial direction, $w_{\perp}\left(\vec{r}-\vec{r}_{i}\right)$, are given by

$$
\begin{aligned}
U= & \frac{4 \pi \hbar^{2} a_{s}}{m} \int d \vec{r}\left|w_{\perp}\left(\vec{r}-\vec{r}_{i}\right)\right|^{4} \int d z\left|w\left(z-z_{i}\right)\right|^{4}, \\
\tilde{U}= & \frac{4 \pi \hbar^{2} a_{s}}{m} \int d \vec{r}\left|w_{\perp}\left(\vec{r}-\vec{r}_{i}\right)\right|^{4}, \\
& \times \int d z\left|w\left(z-z_{i}\right)\right|^{2} w\left(z-z_{i}\right)^{*} w\left(z-z_{i+1}\right), \\
\gamma_{3}= & \Gamma_{3} \int d \vec{r}\left|w_{\perp}\left(\vec{r}-\vec{r}_{i}\right)\right|^{6} \int d z\left|w\left(z-z_{i}\right)\right|^{6} .
\end{aligned}
$$

As before, $a_{s}$ denotes the scattering length, $J$ is the singleparticle nearest-neighbor tunneling amplitude, and $\Gamma_{3}=2 L_{3}$, where $L_{3}$ is the experimentally measured three-body loss parameter [24]. The approximation of restricting the dynamics to $1 \mathrm{D}$ is valid as long as the radial trapping frequency is much larger than the longitudinal trapping frequency, $\omega_{r} \gg$ $\omega_{z}$. Note that here we have taken into account three-body Efimov resonances by incorporating the short distance cut-off term, which has to be introduced to regularize the zero-range three-body pseudopotential $[46,47]$, as a prefactor for a threebody $\delta$-function contact interaction $[12,48]$.

In order to extract the decay rates from this model, we consider four Cs atoms in two lattice sites and we begin with the initial state of two atoms on each site. We then apply a Lindblad master equation,

$$
\frac{d}{d t} \rho=-i\left[H_{\text {eff }}, \rho\right]+2 L \rho(t) L^{\dagger}+2 L_{s} \rho(t) L_{s}^{\dagger},
$$

where $L=\sqrt{\gamma_{3} / 12} b_{i}^{3}$. We have included a single-particle loss rate, $H_{\text {eff }}=H+H_{s}$, where

$$
H_{s}=-i \tilde{S} \sum_{i} b_{i}^{\dagger} b_{i}
$$

and $L_{s}=\sqrt{\tilde{S}} b_{i}$. For the loss rate, we use a value $\tilde{S}=$ $0.006 \mathrm{~Hz}$, which was fine-tuned so that this model would reproduce the lowest experimentally measured value. We then calculate the time-dependent expectation value of the total doublon number in the system and fit an exponential to this function, $\langle\hat{N}(t)\rangle / N(0)=\exp (-\alpha t)$, to extract the decay rate $\alpha$.

Using the master equation we compare two cases: one case where the model coefficients are given by Eq. (C2) with the free space $L_{3}$ parameter [24] and a second case where we include corrections to the on-site lattice parameters due to couplings to higher-energy states induced by two- and three-body interactions. For two-body interactions we make use of the analytical results presented in Ref. [43], and we explain how calculations of the three-body states lead us to corrections for the on-site three-body interaction and also give rise to an effective loss rate in the next section. Note that both calculations approximate each lattice site as an isotropic harmonic oscillator: The value of the energy spacing is given by the geometric mean of the three energies of the anisotropic experiment.

If we set the single particle loss rate, $\tilde{S}=0$, then we can calculate the decay rate through second-order perturbation theory in $J \ll U$,

$$
\Delta E^{(2)}=-2 \frac{|3 \sqrt{6} \tilde{U}-\sqrt{6} J|^{2}}{E_{3 B}\left(a_{s}\right)-2 U_{2 B}},
$$

where $E_{3 B}\left(a_{s}\right)$ and $U_{2 B}$ are the energy shifts due to three and two interacting atoms, respectively. The decay rate is then found through

$$
P(t)=\left\langle\phi\left|e^{i\left(\Delta E^{(2) *}-\Delta E^{(2)}\right) t}\right| \phi\right\rangle=e^{-i 2 \operatorname{Im}\left(\Delta E^{(2)}\right) t},
$$

giving us an expression for the decay rate, $\alpha=2 \operatorname{Im}\left(\Delta E^{(2)}\right)$.

We then use this expression to find the rescaled value for $\tilde{J}=|3 \sqrt{6} \tilde{U}-\sqrt{6} J|$, such that the model (with rescaled on-site coefficients) gives rise to the experimentally observed decay rates. Note that we only perform this fit for larger values of $\left|a_{s}\right|$ (where the perturbation theory is most valid) because for lower values the calculated decay fits the experimental measurements quite well and is dominated by the singleparticle losses.

\section{APPENDIX D: ON-SITE THREE-BODY ENERGY LEVELS AND DECAY}

Our three-body calculations for Cs atoms are performed using the adiabatic hyperspherical representation [31,37], where the interatomic interactions are given by a two-channel model similar to the one used in Ref. [49], which properly describes the $-11-\mathrm{G}$ Feshbach resonance for the $F=3, m_{F}=3$ hyperfine state of Cs [50]. Our methodology, developed in Ref. [51], incorporates the use of Feshbach projectors [52] to represent the fully symmetric three-body spin states as well as the relevant decay channels responsible for atomic 

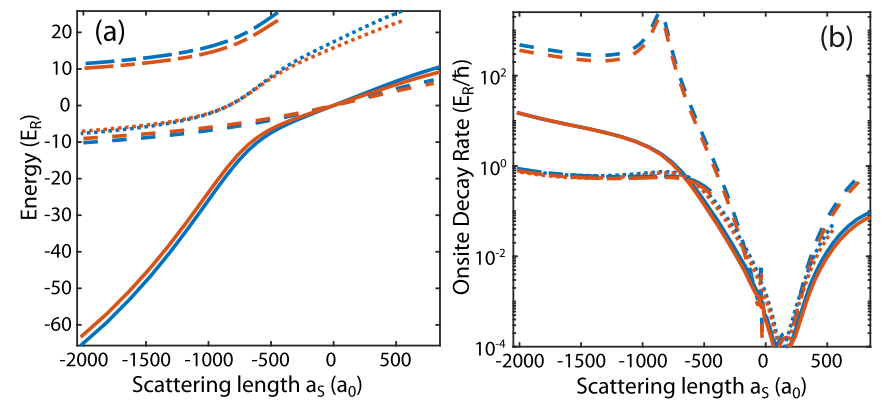

FIG. 4. On-site three-body energies (left) and on-site loss rates (right) calculated using the adiabatic hyperspherical representation [31,37], for $V_{z}=16 E_{R}$ (blue) and $V_{z}=10 E_{R}$ (red). We include the first, second and third three-body state (solid, dotted, dashed-dotted). We also include the (two-body) interaction energy of the initial state of two atoms on each lattice site (left, dashed), calculated using Ref. [43]. And we include the on-site loss rate calculated from the $\mathrm{BH}$ model using the free-space $L_{3}$ parameter [24] (right, dashed).

losses. In the hyperspherical representation the hyperradius $R$ determines the overall size of the system, while all other degrees of freedom are represented by a set of hyperangles $\Omega$. Within this frame work, the three-body adiabatic potentials $U$ and channel functions $\Phi$ are determined from the solutions of the hyperangular adiabatic equation:

$$
\begin{aligned}
& {\left[\frac{\Lambda^{2}(\Omega)+15 / 4}{2 \mu R^{2}} \hbar^{2}+\varepsilon_{\alpha}\right] \Phi_{\alpha}(R ; \Omega)} \\
& +\sum_{\beta} \sum_{i<j} V_{\alpha \beta}\left(r_{i j}\right) \Phi_{\beta}(R ; \Omega)=U(R) \Phi_{\alpha}(R ; \Omega),
\end{aligned}
$$

which contains the hyperangular part of the kinetic energy, expressed through the grand-angular momentum operator $\Lambda^{2}$ and the three-body reduced mass $\mu=m / \sqrt{3}$. In the above equation, our two-channel threshold energies and model potential are given, respectively, by

$$
\varepsilon=\left(\begin{array}{c}
\varepsilon_{\mathrm{bg}} \\
\varepsilon_{\text {res }}
\end{array}\right) \text { and } V(r)=\left[\begin{array}{cc}
V_{\mathrm{bg}}(r) & V_{c}(r) \\
V_{c}(r) & V_{\text {res }}(r)
\end{array}\right]
$$

where the background and resonant interactions are $V_{\mathrm{bg}}(r)=$ $-C_{6} / r^{6}\left(1-\lambda_{\mathrm{bg}}^{6} / r^{6}\right)$ and $V_{\text {res }}(r)=-C_{6} / r^{6}\left(1-\lambda_{\text {res }}^{6} / r^{6}\right)$, with $C_{6}=6890.4768$ being the van der Waals dispersion coefficient for Cs [53], and interchannel coupling $V_{c}(r)=$ $\alpha_{c} \exp \left[-\left(r-\beta_{c}\right)^{2} / 2 \gamma_{c}^{2}\right]$. Assuming that $\varepsilon_{\mathrm{bg}}=0$, we choose the parameters $\lambda_{\mathrm{bg}}, \lambda_{\text {res }}, \alpha_{c}, \beta_{c}, \gamma_{c}$, and $\varepsilon_{\text {res }}$ in order to properly reproduce the magnetic-field dependence of the -11 -G Feshbach resonance. Our calculations are performed assuming $V_{\mathrm{bg}}$ and $V_{\text {res }}$ each containing four $s$-wave molecular states. The values for the energy and corresponding decay are then obtained by solving the hyperradial Schrödinger equation [37] in the presence of a harmonic confinement and leaving the adiabatic channels associated to the diatomic states open, thus allowing the state to decay [36].

The explicit energy and loss values for the lowest and first two excited three-body states are given in Fig. 4. We also include the interaction energy of the initial state in the experiment: two atoms on each lattice site. This allows us to compare the energy shift [see Eq. (C5)] that the system is required to overcome for each three-body state when the
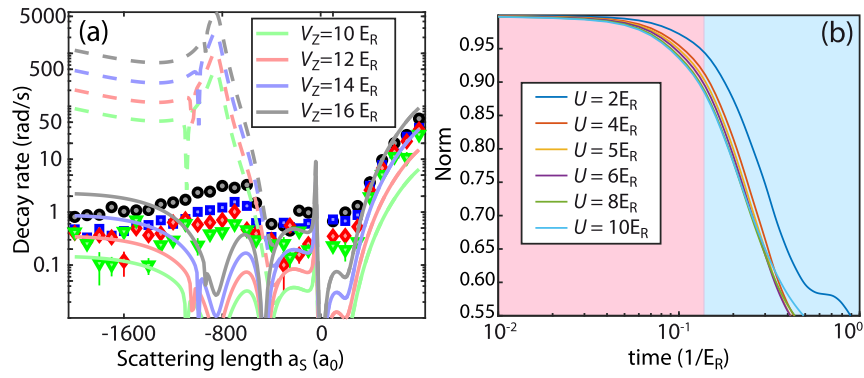

FIG. 5. (a) Comparison of the decay rates predicted from an extended Bose-Hubbard model with only onsite three-body loss (solid), with also including off-site three-body loss between nearest-neighboring lattice sites (dashed) and the experimental measurements (markers). (b) Decay of the normalization for a real space simulation of two particles in a double well with two-body loss. Highlighted is the distinction between two decay regimes, the initial decay that is dominated by offsite processes followed by a second decay region with a different decay rate.

system tunnels from two doubly occupied sites into the triply occupied site, which is the nonresonant process responsible for the atomic loss in the experiment. We can see that for positive and small negative values of $a_{S}$ the three-body state closest to being resonant is the lowest, however, for large and negative values of $a_{S}$, the most resonant state is the first excited one. This may indicate that this is the most likely state that is populated when the third particle tunnels into the site, which leads us to believe that the remaining discrepancies between our theory predictions and the experimental data presented in the main text could be due to couplings into these excited states.

However, the energy resonance condition is not the whole picture, there is still the question of the rescaling of the effective tunneling rates, which complicates this conclusion. And so it is likely that the correct on-site three-body state to use is a nontrivial superposition between the lowest and the first couple of excited three-body eigenstates. We leave the analysis of the coefficients in this superposition as a future objective.

In Fig. 4(b), we can see that for large negative scattering lengths the renormalized values for the onsite decay rates are two orders of magnitude smaller than those calculated with the free space $L_{3}$ parameter [see $\gamma_{3}$ in Eq. (C2)]. This indicates that the onsite losses are too low to induce the quantum Zeno suppression usually expected in this regime.

\section{APPENDIX E: OFF-SITE LOSS}

In Fig. 5(a) we compare the experimental measurements of the doublon decay rates to the decay rates predicted from an extended BH model. As well as the model presented in the main text with only onsite three-body loss, we include results on including in this model a nearest-neighbor three-body loss term,

$$
H_{\text {offsite }}=-i \frac{\gamma_{N N}}{4} \sum_{n} b_{n}^{\dagger 3} b_{n}^{2} b_{n+1}+b_{n} b_{n+1}^{\dagger 3} b_{n+1}^{2}+\text { H.c. },
$$


where

$$
\begin{aligned}
\gamma_{N N}= & \Gamma_{3} \iint d \vec{r}\left|w_{\perp}\left(\vec{r}-\vec{r}_{i}\right)\right|^{6} \\
& \times \int d z\left|w\left(z-z_{i}\right)\right|^{4} w\left(z-z_{i}\right)^{*} w\left(z-z_{i+1}\right) .
\end{aligned}
$$

For positive scattering lengths including off-site loss leads only to small quantitative corrections. However, for negative scattering lengths including off-site loss leads to predictions for the decay rates that are three orders of magnitude larger than the model with only onsite loss and also compared to the experimental results. This indicates that the dominant effect of the renormalization of mixing with higher-energy bands is to suppress these off-site processes.

This can be understood through the following physical picture: We begin the experiment with particles localized in the lowest band Wannier functions on each site. In this initial state, there will be some small overlap between wave functions on neighboring sites. When we then lower the energy barriers in one direction and quench on the interactions and loss terms, then these small overlaps will initially dominate the decay. For strong losses, these overlaps will very rapidly go to zero resulting in new onsite wave functions that are more localized and have a much smaller nearestneighbor overlap and loss coefficient. But in order to obtain more localized wave functions we must mix the lowest band Wannier functions with those from higher bands-which is not captured by the $\mathrm{BH}$ model that we have employed. Meaning that the BH model finds this initial decay rate, whereas we experimentally measure the longer time decay rate where the nearest-neighbor coefficients have been suppressed. This is why the extended $\mathrm{BH}$ model ignoring these off-site coefficients gives results that are closer to those observed experimentally. Off-course, this renormalization will also effect the onsite terms as well as the tunneling coefficients which explains the remaining discrepancy.

In Fig. 5(b) we explicitly assess this physical picture by performing a simulation of atoms in a double well potential, $V_{z}=V_{0} \cos ^{2}(z)$, on a real space grid. This quickly becomes numerically intractable as we increase the number of particles, so we perform a conceptually similar simulation but that is numerically feasible. To this end we consider two bosons in a double well that interact with two-body loss modeled as an imaginary two-body contact interaction. We begin with the initial state with $V_{z}=30 E_{R}$ and $U=0$ where each particle is on each lattice site. We then lower the energy barrier to $V_{z}=10 E_{R}$ and increase $|U|$ and time-dependently calculate the normalization.

From the figure we see that there is a clear distinct behavior between the initial decay and the longer time decay. We have given a rough indication of the separation of these two regimes. Off-course, this cannot be directly compared to the situation happening in the experiment where we believe the effects to be more pronounced. In that case we have two particles initially on each site, there are two-body interactions (as well as three-body loss) which give rise to density assisted tunneling processes between each site. But this analysis gives a clear indication that there is a time-dependent decay rate.

\section{APPENDIX F: FUTURE THEORETICAL ANALYSIS}

In this section we summarize some of the important approximations that go into deriving our models. The purpose of this is to highlight the additional features that should be incorporated for future theoretical studies into the phenomena presented in the main text.

(i) On-site coefficient calculation: This calculation assumes an isotropic harmonic oscillator for a single lattice site, where we have taken the geometrical mean of the energy levels in the three directions in the experimental set-up. The interplay between the anisotropy and the free-space Efimov resonance can allow for additional features on the observed decay rates as displayed in Fig. 3(c).

(ii) Mixing of on-site three-body eigenstates: As shown in Fig. 4, particularly for large and negative values of $a_{S}$, the energy of the initial state (dashed line) is very close to being resonant with the first excited three-body eigenstate, indicating that in this regime there is a strong possibility that these states will also be important and result in a further rescaling of the on-site loss rates. In the main text, we have attempted to predict the values of the renormalized tunneling terms that couple the initial state into a triply occupied site, but for this calculation we ignored the excited three-body eigenstates. One would then need to calculate the individual matrix elements describing the coupling of the initial state into each of the three-body eigenstates separately in order to better predict the overall decay rates.

(iii) Restricting the dynamics to $1 D$ : As stated above, when calculating the effects of strong interactions, which mixes with higher-energy bands, on the effective onsite coefficients, we assume a 3D isotropic lattice. However, when calculating the decay rate induced through the dynamical processes we assume a 1D geometry. The ratio of the tunneling coefficients in the $z$ direction to the radial directions are, $J_{x y} / J_{z}=$ $[0.025,0.038,0.058,0.087]$ for $V_{z}=[10,12,14,16] E_{R}$, and the density assisted tunneling ratio is $U_{x y} / U_{z}<10^{(-5)}$ for all $V_{z}$. This indicates that for the dynamical features 1D is probably a good approximation. However, from Fig. 3(c) the rescaling of the tunneling coefficients is potentially larger for stronger confinement, and so the strong coupling with states in higher bands may be enhancing the single-particle tunneling in the radial directions. Such effects could be reducing the validity of our $1 \mathrm{D}$ approximation, further explaining the discrepancy between the experimental measurements and theoretical calculations.
[1] I. Bloch, J. Dalibard, and W. Zwerger, Rev. Mod. Phys. 80, 885 (2008).

[2] M. Lewenstein, A. Sanpera, and V. Ahufinger, Ultracold Atoms in Optical Lattices: Simulating Quantum Many-Body Systems (Oxford Scholarship Online, Oxford, 2012).
[3] I. Bloch, J. Dalibard, and S. Nascimbène, Nat. Phys. 8, 267 (2012).

[4] M. Müller, S. Diehl, G. Pupillo, and P. Zoller, Adv. At. Mol. Opt. Phys. 61, 1 (2012).

[5] A. J. Daley, Adv. Phys. 63, 77 (2014). 
[6] N. Syassen, D. M. Bauer, M. Lettner, T. Volz, D. Dietze, J. J. García-Ripoll, J. I. Cirac, G. Rempe, and S. Dürr, Science 320, 1329 (2008).

[7] B. Yan, S. A. Moses, B. Gadway, J. P. Covey, K. R. A. Hazzard, A. M. Rey, D. S. Jin, and J. Ye, Nature 501, 521 (2013).

[8] B. Zhu, B. Gadway, M. Foss-Feig, J. Schachenmayer, M. L. Wall, K. R. A. Hazzard, B. Yan, S. A. Moses, J. P. Covey, D. S. Jin, J. Ye, M. Holland, and A. M. Rey, Phys. Rev. Lett. 112, 070404 (2014).

[9] R. Schützhold and G. Gnanapragasam, Phys. Rev. A 82, 022120 (2010).

[10] W. M. Itano, D. J. Heinzen, J. J. Bollinger, and D. J. Wineland, Phys. Rev. A 41, 2295 (1990).

[11] M. J. Gagen, H. M. Wiseman, and G. J. Milburn, Phys. Rev. A 48, 132 (1993).

[12] A. J. Daley, J. M. Taylor, S. Diehl, M. Baranov, and P. Zoller, Phys. Rev. Lett. 102, 040402 (2009).

[13] M. Roncaglia, M. Rizzi, and J. I. Cirac, Phys. Rev. Lett. 104, 096803 (2010).

[14] S. Diehl, M. Baranov, A. J. Daley, and P. Zoller, Phys. Rev. B 82, 064509 (2010).

[15] S. Diehl, M. Baranov, A. J. Daley, and P. Zoller, Phys. Rev. B 82, 064510 (2010).

[16] B. Schmidt, M. Bortz, S. Eggert, M. Fleischhauer, and D. Petrosyan, Phys. Rev. A 79, 063634 (2009).

[17] D. Petrosyan, B. Schmidt, J. R. Anglin, and M. Fleischhauer, Phys. Rev. A 76, 033606 (2007).

[18] Y.-C. Chen, K.-K. Ng, and M.-F. Yang, Phys. Rev. B 84, 092503 (2011).

[19] A. Privitera, I. Titvinidze, S.-Y. Chang, S. Diehl, A. J. Daley, and W. Hofstetter, Phys. Rev. A 84, 021601(R) (2011).

[20] Y.-W. Lee and M.-F. Yang, Phys. Rev. A 81, 061604(R) (2010).

[21] L. Bonnes and S. Wessel, Phys. Rev. Lett. 106, 185302 (2011).

[22] A. Kantian, M. Dalmonte, S. Diehl, W. Hofstetter, P. Zoller, and A. J. Daley, Phys. Rev. Lett. 103, 240401 (2009).

[23] B. Capogrosso-Sansone, S. Wessel, H. P. Büchler, P. Zoller, and G. Pupillo, Phys. Rev. B 79, 020503(R) (2009).

[24] T. Kraemer, M. Mark, P. Waldburger, J. G. Danzl, C. Chin, B. Engeser, A. D. Lange, K. Pilch, A. Jaakkola, H. C. Nägerl, and R. Grimm, Nature 440, 315 (2006).

[25] M. J. Mark, E. Haller, K. Lauber, J. G. Danzl, A. J. Daley, and H.-C. Nägerl, Phys. Rev. Lett. 107, 175301 (2011).

[26] M. J. Mark, E. Haller, K. Lauber, J. G. Danzl, A. Janisch, H. P. Büchler, A. J. Daley, and H.-C. Nägerl, Phys. Rev. Lett. 108, 215302 (2012).

[27] O. Jürgensen, F. Meinert, M. J. Mark, H.-C. Nägerl, and D.-S. Lühmann, Phys. Rev. Lett. 113, 193003 (2014).

[28] K. Winkler, G. Thalhammer, F. Lang, R. Grimm, J. Hecker Denschlag, A. J. Daley, A. Kantian, H. P. Büchler, and P. Zoller, Nature 441, 853 (2006).
[29] N. Strohmaier, D. Greif, R. Jördens, L. Tarruell, H. Moritz, T. Esslinger, R. Sensarma, D. Pekker, E. Altman, and E. Demler, Phys. Rev. Lett. 104, 080401 (2010).

[30] A. Deuchert, K. Sakmann, A. I. Streltsov, O. E. Alon, and L. S. Cederbaum, Phys. Rev. A 86, 013618 (2012).

[31] J. P. D’Incao, J. Phys. B: At., Mol. Opt. Phys. 51, 043001 (2018).

[32] E. Braaten and H.-W. Hammer, Phys. Rep. 428, 259 (2006).

[33] H. P. Büchler, Phys. Rev. Lett. 104, 090402 (2010).

[34] D. Jaksch and P. Zoller, Ann. Phys. 315, 52 (2005).

[35] L.-M. Duan, E. Demler, and M. D. Lukin, Phys. Rev. Lett. 91, 090402 (2003).

[36] A. Goban, R. B. Hutson, G. E. Marti, S. L. Campbell, M. A. Perlin, P. S. Julienne, J. P. D'Incao, A. M. Rey, and J. Ye, Nature 563, 369 (2018).

[37] J. Wang, J. P. D’Incao, and C. H. Greene, Phys. Rev. A 84, 052721 (2011).

[38] V. Efimov, Nucl. Phys. A 210, 157 (1973).

[39] T. Kraemer, J. Herbig, M. Mark, T. Weber, C. Chin, H.-C. Nägerl, and R. Grimm, Appl. Phys. B 79, 1013 (2004).

[40] F. Meinert, M. J. Mark, E. Kirilov, K. Lauber, P. Weinmann, A. J. Daley, and H.-C. Nägerl, Phys. Rev. Lett. 111, 053003 (2013).

[41] A. D. Lange, K. Pilch, A. Prantner, F. Ferlaino, B. Engeser, H.-C. Nägerl, R. Grimm, and C. Chin, Phys. Rev. A 79, 013622 (2009).

[42] D. Jaksch, C. Bruder, J. I. Cirac, C. W. Gardiner, and P. Zoller, Phys. Rev. Lett. 81, 3108 (1998).

[43] T. Busch, B.-G. Englert, K. Rzażewski, and M. Wilkens, Found. Phys. 28, 549 (1998).

[44] J. P. D’Incao, H. Suno, and B. D. Esry, Phys. Rev. Lett 93, 123201 (2004).

[45] G. Thalhammer, K. Winkler, F. Lang, S. Schmid, R. Grimm, and J. H. Denschlag, Phys. Rev. Lett. 96, 050402 (2006).

[46] V. Efimov, Phys. Lett. B 33, 563 (1970).

[47] P. F. Bedaque, H.-W. Hammer, and U. van Kolck, Phys. Rev. Lett. 82, 463 (1999).

[48] P. Naidon and S. Endo, Rep. Prog. Phys. 80, 056001 (2017).

[49] Y. Wang and P. S. Julienne, Nat. Phys. 10, 768 (2014).

[50] C. Chin, R. Grimm, P. Julienne, and E. Tiesinga, Rev. Mod. Phys. 82, 1225 (2010).

[51] R. Chapurin, X. Xie, M. J. Van de Graaff, J. S. Popowski, J. P. D'Incao, P. S. Julienne, J. Ye, and E. A. Cornell, Phys. Rev. Lett. 123, 233402 (2019).

[52] S. Jonsell, J. Phys. B: At., Mol. Opt. Phys. 37, S245 (2004).

[53] M. Berninger, A. Zenesini, B. Huang, W. Harm, H.-C. Nägerl, F. Ferlaino, R. Grimm, P. S. Julienne, and J. M. Hutson, Phys. Rev. A 87, 032517 (2013). 